\title{
Digital simulation of chronopotentiometric and steady-state voltammetric curves at microelectrodes in the presence of a low concentration of supporting electrolyte
}

\author{
Marcin J. Pałys ${ }^{\text {a,b, * }}$, Zbigniew Stojek ${ }^{a}$, Martinus Bos ${ }^{b}$, Willem E. van der Linden ${ }^{b}$ \\ a Laboratory of Theory and Application of Electrodes, Department of Chemistry, University of Warsaw, Pasteura 1, 02-093 Warsaw, Poland \\ ${ }^{\mathrm{b}}$ Laboratory for Chemical Analysis, Department of Chemical Technology, Twente University of Technology, P.O. Box 217 , \\ 7500 AE Enschede, Netherlands
}

Received 5 September 1994

\begin{abstract}
A simulation scheme for the calculation of theoretical chronopotentiograms at microelectrodes in solutions containing low amounts of supporting electrolyte is presented. The scheme allows computation of the changes in the concentration profiles of the substrates, products and the supporting electrolyte ions with time. The electrode potentials that are established after reaching the steady-state, together with the appropriate current intensities, can be used for constructing the steady-state voltammograms. The simulation of the mixed diffusional and migrational transport is based on the Crank-Nicolson method with an exponentially expanding time and space grids. The scheme does not impose any limitations on diffusion coefficients and it can be applied both to simple electrode reactions (one reactant-one product) and more complicated reactions under the assumption that the double-layer thickness is small in comparison to the diffusion layer. Five simple types of electrode reactions and an example of a more complicated scheme were considered. The results obtained demonstrate that the dependence of the steady-state limiting current on the support ratio $\left(c_{\text {supp.el. }} / c_{\text {subst }}\right)$ depends not only on the charge of the reactant and the product, but also on the diffusion coefficient ratio of the substrate and product. If the difference between diffusion coefficients is large, the predictions based on simpler theories available in literature can become invalid.
\end{abstract}

Keywords: Migration; Low ionic strength; Chronopotentiometry; Steady-state voltammetry; Digital simulation

\section{Introduction}

Among the features that triggered the interest in ultramicroelectrodes, small currents and consequently small ohmic drops played an important role [1,2]. Reduction or even removal of the deleterious effect that the $I R$ drop has on voltammetric signals allowed the exploration of domains of electrochemistry inaccessible to the classic approach: measurements at very high concentrations, measurements in resistive solvents or in the presence of very little (or no) supporting electrolyte. Owing to the small $I R$ drop, ultramicroelectrodes have found attractive novel analytical applications [3-7]. Recently, a strong interest was observed in

\footnotetext{
${ }^{*}$ Corresponding author.
}

providing theoretical descriptions of processes taking place under such circumstances [8-16].

A common feature of the above-mentioned situations is that migration plays a significant role and must be taken into account in the transport considerations. This complicates the description, because a change in the charge of the reactant and/or product causes qualitative changes in the observed processes, as compared with only quantitative changes in the purely diffusional transport. The advantage is, however, that the $I R$ drop effect is explicitly considered in the model, so no instrumental ohmic drop correction, based on approximate models, is necessary.

In classic electrochemical work, the effect of migration on the observed currents was derived from the static (time- and current-independent) resistance of the cell. Such treatment is considered inappropriate nowadays $[9,15]$, because it does not account for the redistri- 
bution of ions in the vicinity of the electrode. This effect, caused by the current flow, makes the conductivity dependent on the current intensity. The result of the classic approach was often an over- or underestimation of the $I R$ drop effect in certain classes of situations.

Generally, theories describing electrode processes under conditions of diffusion and migration can be divided into two classes. The first assumes that the thickness of the double layer is much smaller than the thickness of the diffusion (depletion) layer, therefore neglecting the influence of the double layer on the transport. The migrational effects considered originate from the depletion of ions at the electrode surface, associated with the ion fluxes occurring in the diffusion layer to satisfy the electroneutrality condition. This type of theory cannot be applied to extremely low concentrations of the supporting electrolyte and to very small (nanometre size) electrodes. The second class of theories considers the situation when the depletion layer is entirely included in the double layer and the migration resulting from the electric field of the electrode occurs. These theories describe the transport either when the supporting electrolyte is present at a very low level, or when the electrode is very small (diameter in $\mathrm{nm}$ range). These theories sometimes account for the fact that the electric field in the double layer can be large enough to separate positive and negative ions, leading to the violation of the electroneutrality principle.

To distinguish between the domains of applicability of both types of theories, a criterion presented in the paper by Norton et al. [16] can be used. According to this criterion, the depletion layer thickness should be approximately equal to $10 r_{\mathrm{e}}\left(r_{\mathrm{e}}\right.$ is the electrode radius), and the thickness of the double layer can be approximated as $1.5 \kappa^{-1}$, where $\kappa^{-1}$ is the Debye-Hückel length:

$\kappa^{-1}=\sqrt{\epsilon \epsilon_{0} k T / \sum_{i} n_{i} z_{i}^{2} e_{0}^{2}}$

where $n_{i}$ is the number of ions per unit volume and the summation covers all ions in the solution. For a $1: 1$ electrolyte in water, the approximate $\kappa^{-1}$ values for concentrations $10^{-3}, 10^{-5}$ and $10^{-7} \mathrm{M}$ are $100 \mathrm{~nm}$ and $1 \mu \mathrm{m}$, respectively. These thicknesses are much lower if the electrode potential is far from the potential of zero charge. If this criterion is applied to the most widely used sizes of electrodes $\left(r_{\mathrm{e}}\right.$ larger than $1 \mu \mathrm{m}$ ) the double-layer thickness can be neglected if the concentration of the electrolyte is not lower than $10^{-6}$ $\mathrm{M}$. The latter limit of $10^{-6} \mathrm{M}$ is very often that of solutions without deliberately added supporting electrolyte.

The first group of theories (the effects of the double layer are neglected) generally expects that reduction of cations or oxidation of anions should lead to enhancement of the transport of reactant to the electrode, because both migration and diffusion are synergetic $[8,9,13,15]$. Similarly, oxidation of cations or reduction of anions should lead to a decrease in the current because diffusional and migrational transport have opposite directions. In this paper we show that the actual effect may also depend strongly on the ratio of the diffusion coefficients of the reactant and the product.

A number of theoretical treatments for diffusion and migration are available in the literature. Amatore and co-workers $[8,9]$ have developed the equations to describe the quasi-steady-state current at cylindrical electrodes for the case of a fast, uncomplicated electron transfer. Both redox forms were soluble and supporting electrolyte of type 1:1 was present. All diffusion coefficients were assumed to be equal. The authors concluded that (i) in the case of a neutral reactant, the limiting current is not influenced by the level of the supporting electrolyte concentration, (ii) if an electrode reaction of a monovalent ion leads to a neutral product, the limiting current value doubles as the concentration of the electrolyte changes from infinity to zero, and (iii) if the product and the reactant are ions of opposite signs, infinitely large currents should be observed when supporting electrolyte is completely absent (although this case is unlikely to be observed experimentally owing to possible comproportionation reaction $[9,17])$. The way to generalize the results in order to cover other electrode geometries was presented.

In two papers, Oldham presented the theory for the steady-state voltammetry at hemispherical electrodes under conditions of little [10] or no supporting electrolyte [11]. In the first paper, oxidation of ferrocene (uncharged reactant) and reduction of quinone were considered. The second paper dealt with the theory of voltammetry in the absence of supporting electrolyte; it covered all instances of charges of the reactant and the product (except of the case of a neutral reactant) in a simple reaction $\mathrm{R} \rightarrow \mathrm{P}$ ("one reactant-one product"), when both forms are soluble. Baker et al. [14] published a theoretical description of electrodeposition at low levels of supporting electrolyte. The electrode reaction consisted of the neutralization of an ion with formation of an insoluble product.

Experimental studies of the steady-state currents at microdisc electrodes in the absence and in excess of supporting electrolyte were carried out by Cooper and co-workers $[12,13]$ and compared with models developed for microhemispherical electrodes. These authors found relatively good agreement between the theory and experiment in approximately half of the cases studied, whereas for the other cases significant differences were observed.

Recently, an analytical expression for the steady- 
state currents at hemispherical electrodes has been published by Myland and Oldham [15]. This very complete theory presents a solution to the migration-diffusion problem under steady-state conditions, assuming a simple electron transfer step, solubility of the reactant and the product and equality of the diffusion coefficients.

An investigation of electrode processes coupled with a homogeneous chemical step, in low ionic strength solutions under steady-state conditions, was reported recently by Norton et al. [17].

In general, there are three types of limitations in the listed theories: a very simple electrode reaction is assumed, severe restrictions on the diffusion coefficients and applicability to the steady-state only. In most cases a single ion (or molecule) of the reactant is converted into a single ion (or molecule) of the product. To the best of our knowledge, the only exceptions are the mentioned work of Oldham [10], considering quinone reduction, and, to some extent, the situation of so-called migrational current exaltations $[18,19]$, as recently reconsidered by Kharkats and Sokirko [20]. The reason for assuming the above simplifications is that when both the product and the reactant are soluble and have equal diffusion coefficients, and the electrode reaction does not change the number of molecules or ions, the total concentration of all species in each point of the solution is constant and equal to the total bulk concentration $[8,15]$. This greatly simplifies derivation of the equations, but limits the theory to a very small class of situations encountered in reality. Additionally, the treatments $[8-11,15]$ are applicable to the steady state only and do not give any indication of how long it will take to reach the steady state and how the concentrations and electric variables change during that time.

Norton et al. [17] carried out a simulation of chronoamperometry under conditions of the limiting current in order to confirm their conclusions about homogeneous kinetics obtained from the experiment. Although their simulation method was reported to provide time-dependent fluxes and was claimed to be capable of dealing with complicated electrode reactions schemes and with species having different diffusivities, the authors did not provide any details of the calculation scheme or analyse the impact of these factors on their simulation results.

Theories of the second type (depletion layer smaller than the double layer) were presented by Norton et al. [16] and Smith and White [21]. The first applies to very small electrodes or to extremely low concentrations of the supporting electrolyte, when the migration is caused by an unscreened electric field of the electrode. The theory allows the steady-state limiting current to be calculated for the case of simple electrode reaction $\mathrm{R} \rightarrow \mathrm{P}$, as a response to the electrode potential step and shows that under certain conditions, peak-shaped steady-state voltammograms can be obtained, rather than the usual wave-shaped traces. The authors also presented the results of the digital simulation of timedependent limiting fluxes of the participating species in the case of a single-electron reaction leading to a neutral product, under the assumption that the electroneutrality is preserved. However, the description of the simulation scheme presented does not allow a conclusion as to whether the diffusion coefficients were set equal or not.

The paper by Smith and White [21] deals principally with the violation of the electroneutrality principle and the conditions under which this can happen. The theory was based on the solution of the diffusion-migration transport equation and the Poisson equation, and applies to all concentrations of the supporting electrolyte. The results of the digital simulation of the limiting current for a simple electron transfer case (one reactant-one product) under the assumption of equality of diffusion coefficients were presented.

The general limitation of the theories belonging to the second class are the applicability to simple reaction schemes only and the assumptions of equal diffusion coefficients. The particular disadvantage is that they require the value of the zero charge potential of the electrode - a value not always easily available and susceptible to variations when the reactant/electrolyte concentration ratio is varied from $>1$ to $<1$.

The aim of this paper is to present a basic description for both time-dependent and steady-state systems with mixed diffusional and migrational transport. Our goal is to develop a simulation scheme in which all restrictions regarding the values of diffusion coefficients can be relaxed, and electrode reactions that change the total number of ions or molecules in the solution are allowed.

\section{The model}

In our considerations we assume a two-electrode system with a hemispherical working electrode placed on an infinite insulating plane; the counterelectrode is so large and placed so far from the working electrode that its dimensions are not relevant. The solution contains only one electroactive redox system and a supporting electrolyte. The electrode reaction can be written as $m_{1} \mathbf{R}_{1}+m_{2} \mathbf{R}_{2}+\ldots \rightarrow n_{1} \mathrm{P}_{1}+n_{2} \mathrm{P}_{2}+\ldots$, where $\mathbf{R}_{k}$ and $\mathbf{P}_{k}$ are species present in the solution. Supporting electrolyte ions may be involved as reactants in this scheme and also the material of the electrode can be one of the reactants; products may be deposited on the electrode but without changing the shape or the size of the electrode. The reaction occurs in a single step or, if there is a homogeneous process coupled to the electron transfer, its rate is infinitely high. We also assume 
that the double-layer thickness is small compared with the thickness of the diffusion layer, so its contribution to the migrational transport is negligible, and the electroneutrality principle holds at any point in the solution. The diffusion coefficient of each species is constant throughout the solution and the formation of ion pairs is excluded.

\section{Theory}

If a current flows through a solution as a result of an electrode reaction, uncharged molecules are transported by diffusion and ions are transported both by diffusion and migration in the electric field. The Nerns-Planck equation:

$j=-\left(F^{2} / R T\right)(\nabla \Phi) \sum z_{k}^{2} c_{k} D_{k}-F \sum z_{k} D_{k}\left(\nabla c_{k}\right)$

expresses the current density at each point in the solution as a function of the local concentration of all species and the local gradient of the electric field. It is assumed that the Einstein-Planck relationship linking the ion mobility with its diffusion coefficient holds. The transport equation (diffusion and migration) for each species present in the solution has the form

$\left(\partial c_{k} / \partial t\right)=D_{k}\left\{\nabla^{2} c_{k}+z_{k} \nabla\left[c_{k}(F / R T) \nabla \Phi\right]\right\}$

The value of the electric field, $\Phi$, characterizes the resistance of the solution and is macroscopically observed as an ohmic drop associated with the current flow. The magnitude of the ohmic drop can be calculated as ( $\left.\Phi^{\text {surf }}-\Phi^{\text {bulk }}\right)$, and can be determined provided that the current intensity, the ion distribution, and the diffusion coefficients are known. It is important to emphasize that during the electrolysis the ion distribution may change, leading to a change in the resistance. Therefore, predictions of the $I R$ drop based on the static resistance of the cell may become invalid.

Eq. (1) and (2) can be rewritten in spherical coordinates [8]:

$$
\begin{gathered}
I / 2 \pi r_{\mathrm{e}}^{2} n F=-(\partial \Phi / \partial r)(F / R T) \sum_{k} z_{k}^{2} c_{k} D_{k} \\
-\left(\partial c_{k} / \partial r\right) \sum_{k} z_{k} D_{k} \\
\frac{\partial c_{k}}{\partial t}=D_{k}\left\{\frac{\partial^{2} c_{k}}{\partial r^{2}}+\frac{2}{r} \frac{\partial c_{k}}{\partial r}\right. \\
\left.+z_{k} \frac{F}{R T}\left[\frac{2 c_{k}}{r} \frac{\partial \Phi}{\partial r}+\frac{\partial c_{k}}{\partial r} \frac{\partial \Phi}{\partial r}+c_{k} \frac{\partial^{2} \Phi}{\partial r^{2}}\right]\right\}
\end{gathered}
$$

They can be made dimensionless using the following substitutions:

$$
\Psi=(F / R T)\left(\Phi-\Phi^{\text {bulk }}\right)
$$

(dimensionless electric potential) $\tilde{c}_{k}=c_{k} / c_{\text {react }}^{b}$

(dimensionless concentration)

$\tilde{D}_{k}=D_{k} / D_{\text {react }}$

(dimensionless diffusion coefficient)

$\tilde{R}=r / r_{\mathrm{e}}, \tilde{R}_{\max }-r_{\max } / r_{\mathrm{e}}$

(dimensionless distance and simulation space size)

$$
\tilde{T}=t / t_{\max }, t_{\max }=\frac{\left(r_{\max }-r_{\mathrm{e}}\right)^{2}}{N_{x}^{2} D_{\text {react }}}=\frac{\left(\tilde{R}_{\max }-1\right)^{2} r_{\mathrm{e}}^{2}}{N_{x}^{2} D_{\text {react }}}
$$

(dimensionless time)

$$
\tilde{I}=I / 2 \pi r_{\mathrm{e}} n F D_{\text {react }} c_{\text {react }}^{b}
$$

(dimensionless current)

where the concentrations are normalized with respect to the bulk concentration of the reactant, diffusion coefficients are normalized with respect to the diffusion coefficient of the reactant and distances are expressed in units of the electrode radius. Time is expressed as a fraction of the total time of the experiment, i.e. a fraction of the time after which the Nernst diffusion layer, $\sqrt{D t}$, reaches a distance $\tilde{R}_{\mathrm{max}} / N_{x}$ from the electrode. $\tilde{R}_{\max }$ is the size of the simulation space, so concentrations of all species at $\tilde{R}_{\text {max }}$ are equal to their bulk concentrations. $N_{x}$ was usually taken as 6 [22].

The above substitutions, combined with the following transformation rules for the derivatives:

$$
\begin{aligned}
& \frac{\partial f}{\partial r}=\frac{1}{r_{\mathrm{e}}} \frac{\partial f}{\partial \tilde{R}} \\
& \frac{\partial^{2} f}{\partial r^{2}}=\frac{1}{r_{\mathrm{e}}^{2}} \frac{\partial^{2} f}{\partial \tilde{R}^{2}}
\end{aligned}
$$

and

$$
\frac{\partial f}{\partial t}=\frac{1}{t_{\max }} \frac{\partial f}{\partial \tilde{T}}=\frac{D_{\text {react }} N_{x}^{2}}{\left(\tilde{R}_{\max }-1\right)^{2} r_{\mathrm{e}}^{2}} \frac{\partial f}{\partial \tilde{T}}
$$

yield the equations that form the basis of the simulation:

$$
\begin{aligned}
\frac{\partial \Psi}{\partial \tilde{R}}= & \frac{-1}{\sum_{k} z_{k}^{2} \tilde{c}_{k} \tilde{D}_{k}}\left[\frac{1}{\tilde{R}^{2}} \frac{i}{2 \pi r_{\mathrm{e}} n F D_{\text {react }} c_{\text {react }}^{b}}\right. \\
& \left.+\sum_{k} z_{k} \tilde{D}_{k}\left(\frac{\partial \tilde{c}_{k}}{\partial \tilde{R}}\right)\right] \\
\frac{\partial \tilde{c}_{k}}{\partial \tilde{T}}= & \tilde{D}_{k} \frac{\left(R_{\max }-1\right)^{2}}{N_{x}}\left[\frac{\partial^{2} \tilde{c}_{k}}{\partial \tilde{R}^{2}}+\frac{2}{\tilde{R}} \frac{\partial \tilde{c}_{k}}{\partial \tilde{R}}\right. \\
& +z_{k}\left(\frac{2 \tilde{c}_{k}}{\tilde{R}} \frac{\partial \Psi}{\partial \tilde{R}}+\frac{\partial \tilde{c}_{k}}{\partial \tilde{R}} \frac{\partial \Psi}{\partial \tilde{R}}\right. \\
& \left.\left.+\tilde{c}_{k} \frac{\partial^{2} \Psi}{\partial \tilde{R}^{2}}\right)\right]
\end{aligned}
$$


Our goal is to obtain both time-dependent and steady-state concentration profiles, and also the pairs of the corresponding current-potential values under these two classes of circumstances. To reach this goal, two approaches are possible: simulation of chronoamperometry and simulation of chronopotentiometry. The first approach is rather complex, because to calculate the concentrations of the reactant and the product, the potential imposed on the electrode must be corrected for the $I R$ drop. However, the $I R$ drop depends on the concentrations of all species and on the current magnitude, which are not known yet. Therefore, to obtain the current and potential values for each single time step, a number of iterations must be done. The only exception is the simulation of the limiting current if one assumes that an unspecified potential, large enough to reach the limiting current and to overcome the $I R$ drop, is imposed on the electrode [17].

The simulation of chronopotentiometry is much simpler. Since the current magnitude is known in advance, the electric field gradient $\partial \Phi / \partial R$ (and the $I R$ drop) can be calculated directly and used in the calculations of the new concentration profiles. The new concentrations can then be used to calculate the electrode potential.

The chronopotentiometric approach also has an extra advantage: the type of the electron transfer need not to be specified for the simulation of the concentration profiles. The actual potential of the electrode can be calculated using the surface concentrations of the species, the $I R$ drop value, $\Phi^{\text {surf }}-\Phi^{\text {bulk }}$, and an expression relating $c^{\text {surf }}$ to $E$. Because only the latter relation depends on the electrode kinetics, the simulation need carried out only once to yield the concentration profiles for reversible, quasi-reversible and irreversible systems.

To carry out the simulation of a chronopotentiometric experiment, the following boundary and initial conditions have to be defined:

$\left.\frac{\partial \tilde{c}_{k}}{\partial \tilde{R}}\right|_{\tilde{R}=1}+\left.z_{k}\left(\tilde{c}_{k} \frac{\partial \psi}{\partial \tilde{R}}\right)\right|_{\tilde{R}=1}= \pm \frac{\tilde{I}}{\tilde{D}_{k}}$

( + for reactant, - for product)

$\left.\frac{\partial \tilde{c}_{k}}{\partial \tilde{R}}\right|_{\tilde{R}=1}+\left.z_{k}\left(\tilde{c}_{k} \frac{\partial \Psi}{\partial \tilde{R}}\right)\right|_{\tilde{R}=1}=0$

(for non-electroactive species)

$\tilde{c}_{k} \mid \bar{R}_{=} \bar{R}_{\max }=\tilde{c}_{k}^{\text {bulk }}$

$\left.\tilde{c}_{k}\right|_{(\tilde{T}=0)}=\tilde{c}_{k}^{\text {bulk }}$

the term $I / 2 \pi r_{\mathrm{e}} n F D_{\text {react }} c_{\text {react }}^{\mathrm{b}}$ in Eq. (7) defines the dimensionless current, $I$. Eq. (7) is valid for a simple reaction such as

$R^{z_{\mathrm{R}}} \rightarrow P^{z_{\mathrm{P}}}+\left(z_{\mathrm{P}}-Z_{\mathrm{R}}\right) \mathrm{e}$
For more complicated reactions, e.g.

$m_{\mathrm{A}} \mathrm{A}+m_{\mathrm{b}} \mathrm{B} \stackrel{n \mathrm{e}^{-}}{\longrightarrow} m_{\mathrm{C}} \mathrm{C}+m_{\mathrm{D}} \mathrm{D}$

the boundary condition equations for species $\mathrm{A}, \mathrm{B}, \mathrm{C}$ and $\mathrm{D}$ will have the form

$\left.\frac{\partial \tilde{c}_{k}}{\partial \tilde{R}}\right|_{\tilde{R}=1}+\left.z_{k}\left(\tilde{c}_{k} \frac{\partial \Psi}{\partial \tilde{R}}\right)\right|_{\tilde{R}=1}= \pm m_{k} \frac{\tilde{I}}{\tilde{D}_{k}}$

with the + sign applicable to species $A$ and $B$ and the - sign to C and D. If one of the reactants is the material of the electrode or if one of the products is deposited on the electrode, its concentration profile need not to be simulated. The simulation scheme described remains valid under the assumption that the shape and size of the electrode do not change with time.

Finally, because of neglecting the double-layer effects, the electroneutrality condition $\sum c_{k} z_{k}=0$ at each point in the solution should be obeyed. This condition indirectly expresses the coupling of the fluxes of charged species in the solution, making the transport of anions dependent on the transport of cations. Such a coupling becomes particularly important when diffusion coefficients of oppositely charged species differ significantly.

\section{Simulation method}

The simulation of a concentration profile for each time increment is done in two steps: first, the electric field gradient $\partial \Psi / \partial \tilde{R}$ is calculated for each grid point on the basis of the normalized current value $\tilde{I}$ and the actual concentrations of all species (Eq. (5)). This gradient is then inserted into the differential Eq. (6) for each species and the concentration profile is obtained by solving this equation using the Crank-Nicolson method [23]. The boundary conditions (7)-(11) are used to modify the first and the last row of the tridiagonal matrix obtained in this method. A well known problem with the classical Crank-Nicolson scheme is the difficulty of dealing with boundary conditions in the derivative form, e.g. Eq. (7). To overcome this problem, an implicit form of this condition proposed originally by Heinze et al. [24] was used in the way advocated by Britz [22].

To satisfy the electroneutrality condition the newly obtained concentrations are examined to detect the possible charge excess in any point. If an excess is present, it is distributed among the ions so that the corrections to the cation concentrations compensate half of the excess and the corrections to the anion concentrations the other half. The corrections to individual concentrations can be either proportional to changes in these concentrations in the considered time interval or proportional to the bulk concentrations of the considered species. The first approach is more 
correct, but it is very sensitive to instabilities of the obtained solutions and actually induces and enhances oscillations. Therefore, we decided to use the second approach, which is more approximate but free from the instability effects.

The procedure described assumes implicitly that the change in the electric gradient with change in the local concentrations within a time interval is negligible, which for some cases requires the use of very small time steps and grid intervals.

\subsection{Grids and intervals}

An exponentially expanding space grid was used with the increments increasing according to the modified Feldberg function [25] $\rho=\ln [1+a(\tilde{R}-1)]$ (parameter $a$ was in the range $2-3$ ). The time increment principally increased according either to the power function $\delta \bar{T}=\delta \tau^{\mathrm{p}}$ ( $p$ in the range 2-3) or to the exponential function $\delta \tilde{T}=\exp (a \delta \tau)(a=2-3)$, unless the system was likely to begin to oscillate. These two transformations reduced drastically the number of grid points and time steps necessary to approach the steady state. Usually, the number of grid points employed was a few hundred, and the number of time increments used to simulate the steady state could even be as low as a few tens.

As already mentioned, too large a time increment can cause oscillations. However, finding an optimal value of this increment is only a matter of trials. In our scheme we adopted a strategy in which the time increment is subdivided when the change in the concentration of any species is larger than $75 \%$ of its value in any point of the grid. The size of subintervals is adjusted in such a manner that the expected concentration change during the subinterval should not exceed $75 \%$ and reduced further when necessary.

If the change in the concentration exceeds $75 \%$, irrespective of how small the subintervals are, or if a negative concentration (for one or more species) is obtained, irrespective of the length of the time increment used, the steady state cannot be reached. This generally indicates that the current exceeds the maximum possible flux of the electroactive compound.

If the simulation of a chronopotentiometric experiment is carried out for a long time, the system approaches the steady state, and the steady-state potential corresponding to the applied electrode current can be calculated. For the simplest case of the fast and uncomplicated ("reversible") electron transfer, the electrode potential can be obtained from the relation

$$
\begin{aligned}
& E-E^{\circ \prime}=(R T / n F) \ln \left(c_{\text {prod }}^{\text {surf }} / c_{\text {react }}^{\text {surf }}\right)+\Psi_{\bar{R}=1} \\
& \Psi_{\tilde{R}=1}=\int_{\tilde{R}=1}^{\bar{R}=\infty}(\partial \Psi / \partial \tilde{R}) d \bar{R}
\end{aligned}
$$

According to the definition of $\Psi, \Psi^{\infty}=\Psi^{\text {bulk }}=0$.
If other types of electrode kinetics are considered, appropriate expressions to calculate the potential can used, e.g.

$E-E^{\circ \prime}=(R T / \alpha n F) \ln \left(c_{\text {react }}^{\text {surf }} K / 2 \tilde{I}\right)+\Psi_{\bar{R}=1}$

$K=2 k^{0} r_{\mathrm{e}} / D_{\text {react }}$

in the case of completely irreversible reduction process (see also Ref. [15]).

\subsection{Capabilities and limitations of this simulation scheme}

Generally, the described simulation procedure allows one to obtain three types of results: (i) concentration profiles for a given current density, (ii) chronopotentiometric curves and (iii) steady-state $I-E$ curves. The last can be produced by carrying out a number of simulations for various current densities and collecting the final steady-state current-potential pairs. In this way one can obtain results identical with those derived for steady-state voltammograms by other workers $[8,9,15]$. The simulated concentration profiles are unconditionally valid while the shapes of chronopotentiograms and steady-state $I-E$ curves depend on the rate of the electron transfer.

The simulation method described can be applied to any reaction of the type

React $1+$ React $2+\ldots \stackrel{n \mathrm{e}^{-}}{\longleftrightarrow}$ Prod $1+\operatorname{Prod} 2+\ldots$

The method does not need any assumption about the values of the diffusion coefficients. It is also irrelevant whether the reactants or products are soluble or not. In addition, the scheme can be used, without any modification, to simulate linear current sweep $(I=f(t))$ potentiograms.

\section{Software}

The simulation program was written in the $\mathrm{C}$ programming language (ANSI C) to ensure its portability. The program consists of a main routine carrying out the simulation of the time-dependent concentration profile. This routine is accompanied by a number of procedures to control the correctness of the input data, to calculate the electrode potential, to provide the Myland-Oldham solution (if applicable), and by the user interface. The program was compiled and tested on a number of computer platforms, including IBM PC 486, Telmat (Motorola mc 88100 processor) and IBM AIX (R6000 processor) workstations and CRAY YEL98 and CONVEX C-240 computers.

In many situations, when the local concentrations differ only slightly from their bulk values, subtraction or addition of concentrations can cause a significant loss of precision. Therefore, instead of the local concentration the differences $\bar{c}_{k}-\tilde{c}_{k}^{\text {bulk }}$, which are close to 
zero and more precisely represented by the computer, were employed. To enhance the numerical precision further, the solution of the tridiagonal system of equations, produced by the Crank-Nicolson method, was refined using the so-called iterative improvement method [26].

\section{Validation}

The simulation procedure and the program were validated by comparing the simulation results with analytical equations of Mamantov and Delahay [27] (purely diffusional transport to a hemispherical electrode):

$$
\begin{aligned}
\tilde{c}_{\text {react }}^{\text {surf }}= & 1-\tilde{I}\left\{1-\exp \left[(\tilde{R} \max -1)^{2} \tilde{T} / N_{x}^{2}\right]\right. \\
& \left.\times \operatorname{erfc}\left[(\tilde{R} \max -1) \sqrt{\tilde{T}} / N_{x}\right]\right\}
\end{aligned}
$$

and of Myland and Oldham [15] (diffusion and migration to a hemispherical electrode). An excellent agreement with the Mamantov and Delahay theory was obtained when migrational effects were neglected in the simulation: the differences between the predicted and the simulated concentrations were less than $0.1 \%$. Only for very short times were the differences higher, but they could be reduced by decreasing the time increment and/or the grid interval.

According to Myland and Oldham [15], seven classes of situations can be encountered when a reaction $\mathrm{R}^{z_{\mathrm{R}}}$ $\rightarrow \mathbf{P}^{z_{\mathrm{P}}}+\left(z_{\mathrm{P}}-z_{\mathrm{R}}\right) \mathrm{e}^{-}$(both forms are in the solution) is considered. In our paper we limit this number to five: charge production, charge cancellation, charge increase, charge decrease and sign reversal (definitions are given in Table 1).

The theories of Myland and Oldham [15] and of Amatore and co-workers [8,9] predict that for the charge production reactions the current intensity does not depend on the support ratio (SR), i.e. the ratio of the concentrations of supporting electrolyte and the reactant. The charge cancellation reactions should lead to an increase of the current with a decrease of the SR; the limiting current in the total absence of supporting electrolyte should be twice as large compared with

Table 1

Definition of classes of electrode reaction of type $\mathrm{R}^{z_{\mathrm{R}}} \rightarrow \mathrm{P}^{z_{\mathrm{P}}}+\left(z_{\mathrm{P}}-\right.$ $\left.z_{\mathrm{R}}\right) \mathrm{e}$

\begin{tabular}{ll}
\hline Class name & Definition \\
\hline Charge cancellation $^{\mathrm{a}}$ & $z_{\mathrm{R}} \neq 0, z_{\mathrm{p}}=0$ \\
Charge decrease & $\left|z_{\mathrm{R}}\right|>\left|z_{\mathrm{P}}\right|>0$ \\
Charge increase & $0<\left|z_{\mathrm{R}}\right|<\left|z_{\mathrm{P}}\right|$ \\
Charge production $^{\mathrm{b}}$ & $z_{\mathrm{R}}=0, z_{\mathrm{P}} \neq 0$ \\
Sign reversal & $\operatorname{sgn}\left(z_{\mathrm{R}}\right) \neq \operatorname{sgn}\left(z_{\mathrm{P}}\right)$ \\
\hline
\end{tabular}

ancluding the case of deposition on electrode.

b Including the case of dissolution of the electrode material.
Table 2

The set of simulation parameters chosen for further calculations

\begin{tabular}{ll}
\hline$\tilde{R}_{\max }=1200$ & $\delta \tilde{R}=0.01$ \\
& $\delta \tilde{T}=1 \times 10^{-9}$ \\
$N_{x}=6$ & $\tilde{T}$ transform: $\tau=\ln (1+a \tilde{T}), a=0.5$ \\
Step in $\tilde{I}=0.01$ & $\tilde{R}$ transform: $\rho=\ln [1+a(\tilde{R}-1)], a=3$ \\
\hline
\end{tabular}

$\mathrm{SR}=\infty$. When the absolute value of the charge increases during the electrode reaction, the current drops, while the reverse effect is observed in charge-decrease reactions. The sign reversal is a special case: when the supporting electrolyte concentration decreases, the current increases, reaching an infinite value when no electrolyte is present. This increase is accompanied by a change of shape of the steady-state $I-E$ curve from a wave to a ramp.

To validate our simulation method, a number of series of steady-state $I-E$ curves (reversible electron transfer) were simulated for each class and compared with the Myland-Oldham predictions. Within each series, the simulations were carried out for various support ratios. The simulation parameters (size of the simulation space, time interval, grid interval, time and space transformations) differed in each series. On the basis of these simulations we chose a set of conditions (Table 2), which is a trade-off between the precision and the calculation time.

The decrease of the simulation space size slightly aggravated the results, whereas an increase of the grid intervals close to the electrode had a very clear negative effect on the quality of the results. The impact of the variation of the time interval and the change of the time transformation function (parameter $p$ ) on deviations from the Myland-Oldham model is moderate and lacks a clear trend. A decrease of parameter $a$ in the transformation function for the space slightly improves the results, at the significant expense of the calculation time and the memory requirements.

Fig. 1 presents the deviations of the simulated steady-state $I-E$ curves from the Myland-Oldham predictions, obtained with the set of parameters from Table 2, and expressed in terms of the limiting current, $I_{\text {lim }}$, and half-wave potential, $E_{1 / 2}$, for various classes of the electrode reactions (nernstian charge transfer assumed). It can be seen that these deviations are fairly small (ca. 1\%) if the support ratio is higher than 0.1 . Below this value, the simulation for the charge increase and charge decrease reactions goes fairly well, but it is slightly worse for the charge cancellation - the error becomes larger, up to ca. $8 \%$ for SR lower than $10^{-4}$. In the case of the charge production and sign reversal reactions, reliable $I_{\lim }$ and $E_{1 / 2}$ values can be obtained only for the support ratios $>0.01$, because for lower support levels the oscillations at high current intensi- 

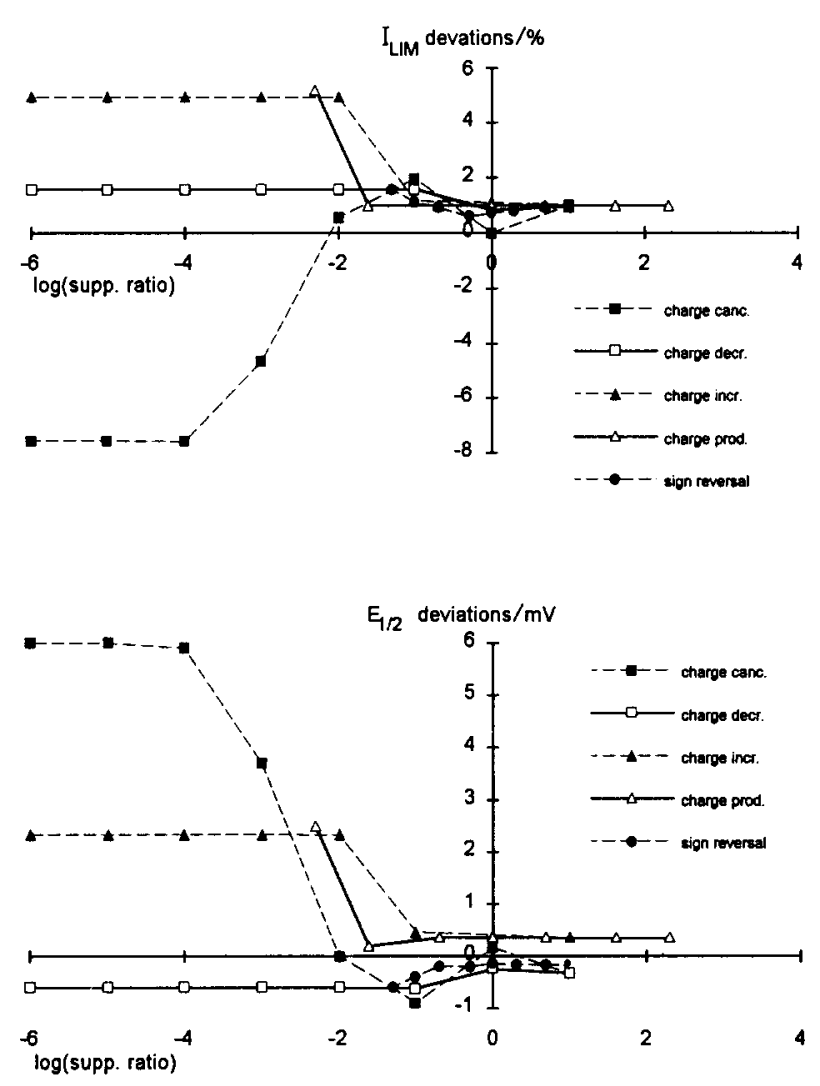

Fig. 1. Differences between simulated steady-state $I-E$ curves and Myland-Oldham predictions, under the chosen simulation conditions: limiting current and half-wave potential.

ties do not allow $I_{\text {lim }}$ (and consequently $E_{1 / 2}$ ) to be determined, so only part of the $I-E$ curve can be obtained.
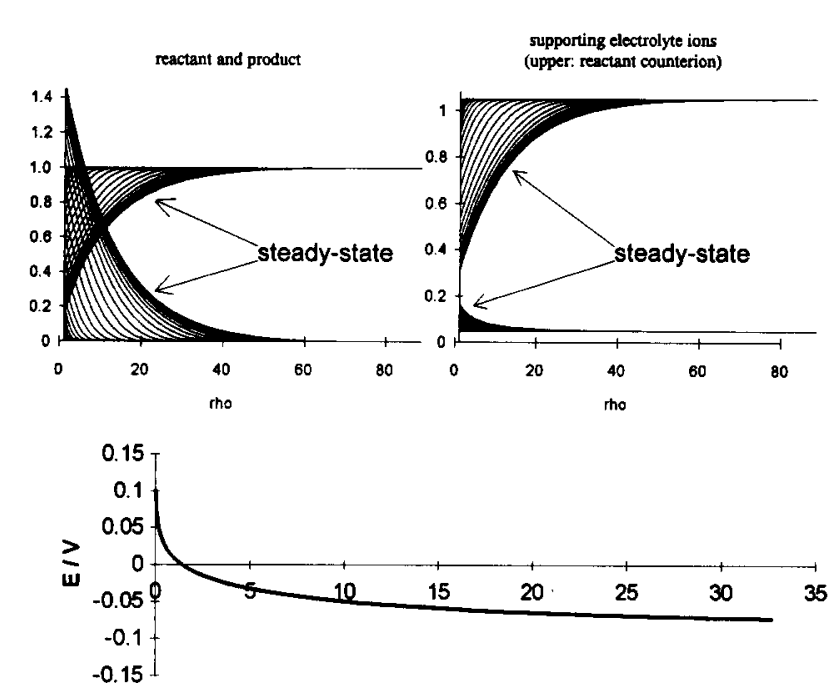

$10^{-3} \times$ square root of normalized time

Fig. 2. Concentration profiles and chronopotentiogram for the electrode reaction with charge cancellation. The abscissa of profiles is the transformed dimensionless distance, $\rho=\ln [1+3(R-1)]$. Simulation conditions: $R_{\max }=1200$, first $\delta \rho=0.01, \delta \mathrm{T}=1.0 \times 10^{-9}, N_{x}=6$, $I=0.8$, all diffusion coefficients equal, $\tilde{c}_{\text {supp }}=0.05$.
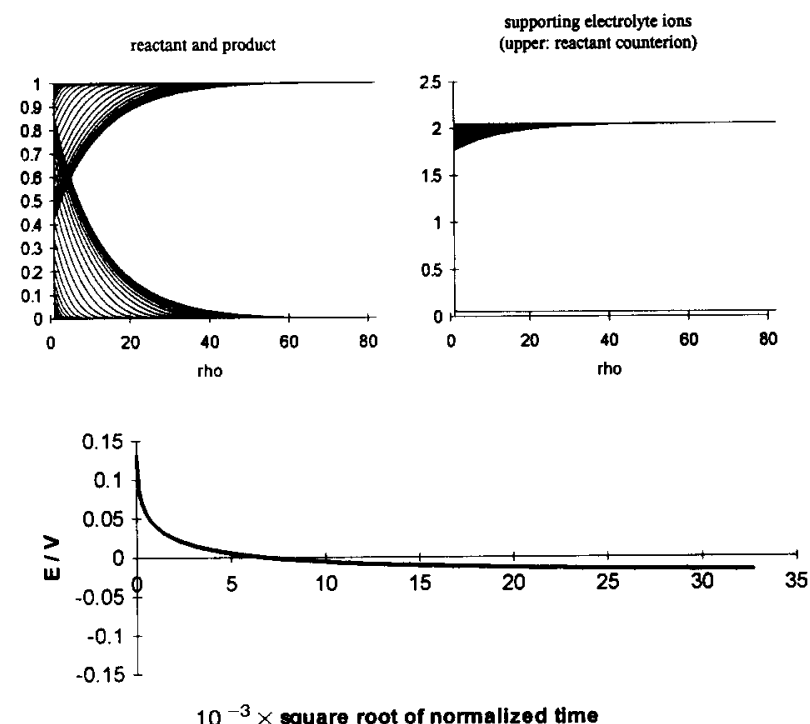

Fig. 3. Concentration profiles and chronopotentiogram for the electrode reaction with charge decrease. Simulation conditions as in Fig. 2.

Closer examination of situations when the calculated concentration profiles start to oscillate suggests that in a number of cases the forcing of electroneutrality may be an origin of instabilities. As already mentioned, the way in which the weighting factors for the distribution of the excess of charge are calculated is approximate, and it can be responsible for distorting the concentration profile, followed by oscillations. The improvement of excessive charge distribution algorithm would probably reduce the observed deviations from the Myland-Oldham model for low values of the support ratio.

It should be noted that the errors in $E_{1 / 2}$ depend on errors in $I_{\text {lim }}$, which can be clearly seen in Fig. 1: if the latter is not exactly known, the former is determined wrongly. Because in some cases oscillations deteriorate the concentration profiles at currents close to the limiting value, for such systems the half-wave potential cannot be obtained. The differences in the performance of the presented simulation scheme can be understood when an analysis of the time-dependent concentration profiles is made. This will be discussed in the following section.

\section{Results and discussion}

\subsection{Time-dependent concentration profiles}

Figs. 2-6 present a series of the concentration profiles, simulated for an exponentially expanding time grid, and for five different electrode processes. The simulation conditions are identical; the current is close to $80 \%$ of the limiting current in each case. In each 

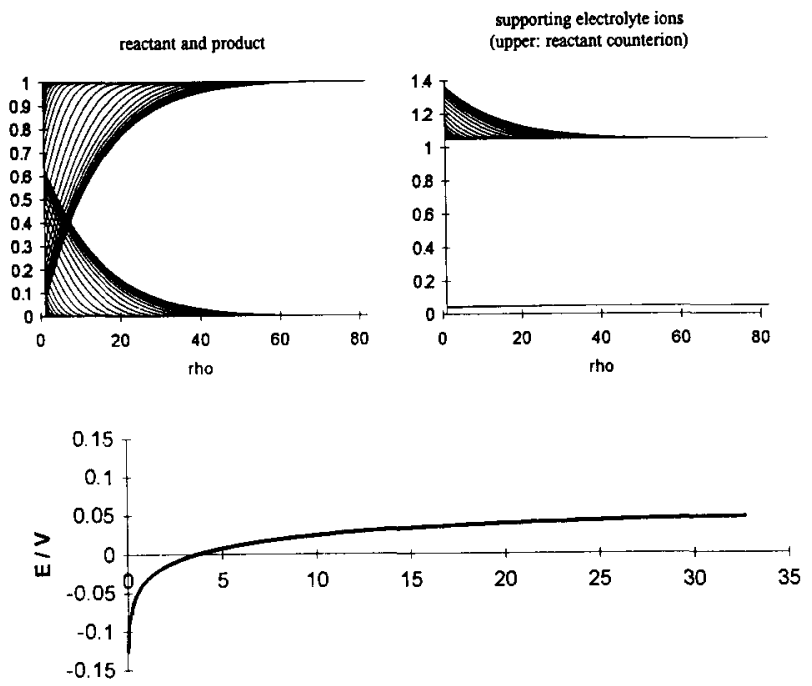

$10^{-3} \times$ square root of normalized time

Fig. 4. Concentration profiles and chronopotentiogram for the electrode reaction with charge increase. Simulation conditions as in Fig. 2.

figure the concentration profiles for the reactant and the product and also for the cation and the anion of the supporting electrolyte are shown. They are accompanied by the chronopotentiogram constructed under the assumption of the Nernstian charge transfer and equality of diffusion coefficients of all species (including supporting electrolyte ions). Figs. 7 and 8 show concentration profiles obtained for the reaction with the product deposited on the electrode and the reaction in which the material of the electrode is the reactant.
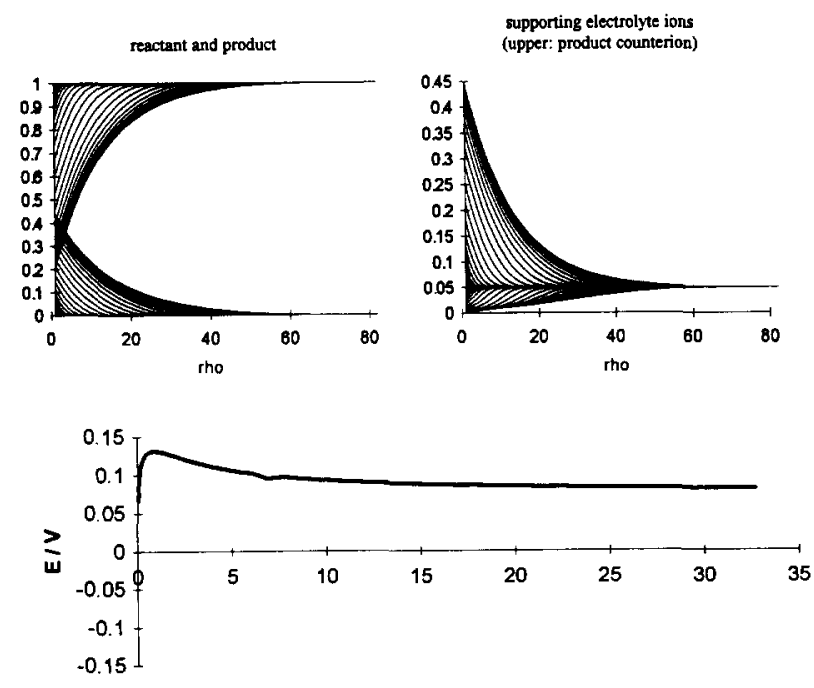

$10^{-3} \times$ square root of normalized time

Fig. 5. Concentration profiles and chronopotentiogram for the electrode reaction with charge production. Simulation conditions as in Fig. 2.
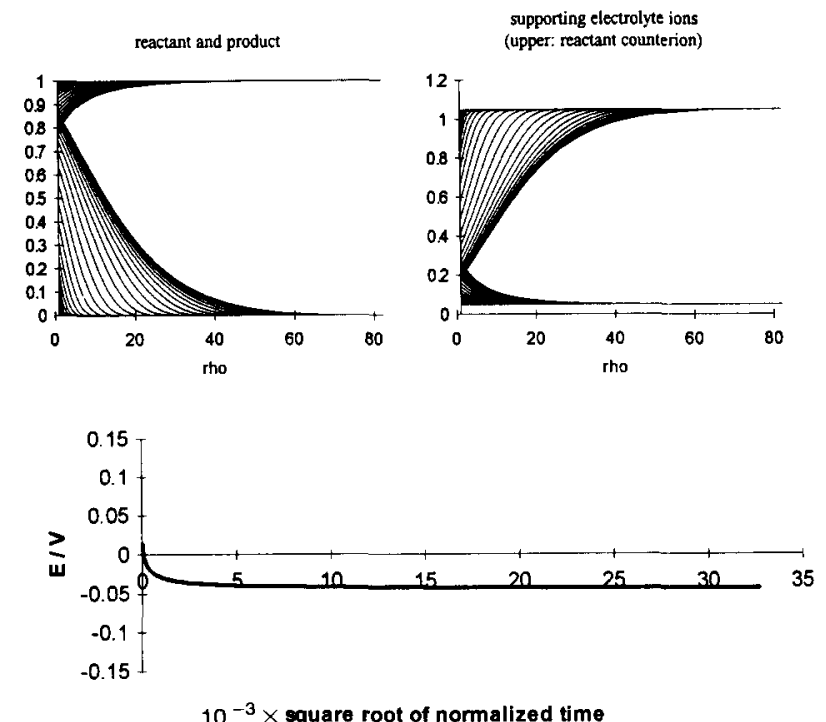

Fig. 6. Concentration profiles and chronopotentiogram for the electrode reaction with sign reversal. Simulation conditions as in Fig. 2.
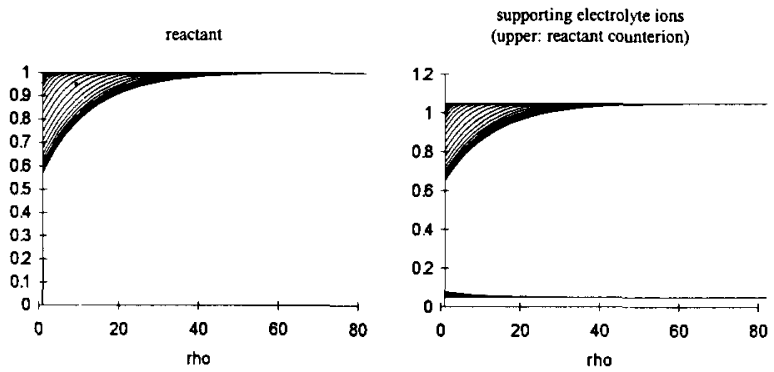

Fig. 7. Concentration profiles for the electrode reaction with product deposited on the electrode. Simulation conditions as in Fig. 2.

A comparison of the concentration profiles of the supporting electrolyte components shows how important the effect of migration can be. In the absence of migration the concentration of supporting electrolyte should be constant at each point of the solution. Analysis of the figures can also reveal the reasons for the simulation problems of certain classes of steady-state $I-E$ curves.
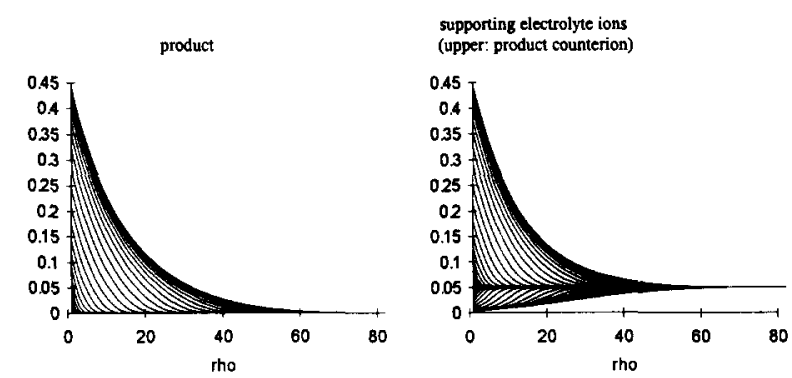

Fig. 8. Concentration profiles for the reaction in which electrode material is a reactant and the product is soluble. Simulation conditions as in Fig. 2. 
The first case, illustrated in Fig. 2, is an example of a charge cancellation reaction. Generally, in this type of reaction the reactant is attracted to the electrode, while a neutral product is transported only by means of diffusion. To maintain the electroneutrality, the counterion of the reactant is repelled from the electrode, leading to a very characteristic increase of the solution resistance at low support ratios. If the concentration of the supporting electrolyte is set to zero, then under the condition of the limiting current the concentration of the reactant is zero and so is the concentration of its counterion; the solution near the electrode has an infinite resistance and the value of the electric field gradient also becomes infinitely large. The increase of $\partial \Psi / \partial R$ with time leads to computational problems: the usual increase of the time interval, combined with the change in electric gradient, makes the simulation inaccurate, which is reflected by lower performance of the simulation scheme when the supporting electrolyte concentration is low.

The cases of charge decrease and increase (Figs. 3 and 4) do not show any peculiarities. The reactant is attracted or repelled from the electrode, respectively, and the product is always repelled (equal diffusion coefficients are assumed). The changes in the concentration profiles of the supporting electrolyte components are typical: the concentration of the ion that carries a charge opposite to that of the product increases significantly.

The case of charge production is the only situation when the transport of the reactant is not influenced by the migration and is thus independent on the support ratio. However, when the concentration of the electroactive component is large compared with the concentration of the supporting electrolyte, large currents flow but the resistance of the solution is high. This results in high gradients of the electric field (the $I R$ drop can reach kilovolts) and causes computational problems. These gradients decrease rapidly with time because the product of the electrode reaction is an ion and because of the electroneutrality principle the ions of supporting electrolyte (for product neutralization) are attracted from the solution. The turning point of the potential, that can be observed on the chronopotentiogram, illustrates the above explanation: initially, the potential value is mainly determined by the $I R$ drop, then the ohmic drop decreases and the change of the surface concentrations of the reactant and the product are responsible for its further variation.

The fact that the simulation performance decreases for low support ratios is not a very serious problem, because such a situation is not easily encountered in real situations: the value of the current in the initial part of the chronopotentiogram would show a transient from zero to the target value.

In the last case (sign reversal) the reactant is at- tracted to the electrode, and the product is repelled. Migration thus enhances the transport of both species. The migrational effect is so large that the concentration profile of the reactant is levelled and changes very little with increase of the current. In the example shown (Fig. 6), the main process in the solution is the replacement of the supporting electrolyte ions by the reaction product: the zone adjacent to the electrode surface consists almost exclusively of reactant and product, whereas the distant part of the solution consists of the reactant and its counterion.

Such a situation is inconvenient from the point of view of the calculations: the concentration profile of the counterion contains a flat segment (very low concentration) close to the electrode, with a very small concentration gradient. Small imperfections in the calculations of concentration at a point in this part of the profile easily lead to large changes of the concentration in the surrounding points, giving rise to oscillations that yield negative concentrations.

Figs. 7 and 8 show concentration profiles obtained for the cases of deposition of the product on the electrode surface and dissolution of the electrode material, respectively. These profiles are similar to those in Figs. 2 and 5. In the construction of these profiles it was assumed that the size and shape of the electrode remain constant during the process.

\subsection{Steady-state I-E curves for simple electrode reac- tions}

The main result of this study is a complete picture of the dependence of parameters of steady-state $I-E$ curves (limiting current and half-wave potential) on the support ratio and on the ratio of diffusion coefficients of the product and reactant (the diffusion coefficients of supporting electrolyte ions were assumed to be equal to the diffusion coefficient of the reactant). Three examples (presented in Figs. 9-11) illustrate this for reactions with a charge increase, a charge decrease and charge cancellation. Such types of dependences cannot be obtained from any of the existing theories, which assume equality of the diffusion coefficients of the reactants and products.

Fig. 9 shows that a change in the diffusion coefficients can influence drastically the steady-state currents: according to the predictions of the MylandOldham theory, the magnitude of the limiting current should decrease with a decrease in the supporting electrolyte concentration. Our results show that this decrease can even be enhanced if the diffusion coefficient of the product is relatively small (accumulation of the reaction product takes place in the vicinity of the electrode). However, for a high ratio of the diffusion coefficients this trend can be reversed and the current actually increases with a decrease in the support ratio. 
The latter effect can be explained by very fast transport of the product from the electrode to the bulk of the solution. The removal of highly charged positive ions from the electrode causes the inversion of the electric gradient in the solution and, as a result, the transport rate of the singly charged reactant increases. Although such a situation (a product with a higher charge and a higher diffusion coefficient) is not likely to be encountered, our results will also apply to any reaction of the type $\mathrm{R}^{z_{\mathrm{R}}} \stackrel{n^{e}}{\leftrightarrow} \mathrm{P}_{1}^{z_{\mathrm{P}}}+\mathrm{N}_{1}^{0}+\mathrm{N}_{2}^{0}+\ldots$, where more products are formed, all but one neutral. Products $\mathbf{N}_{i}$ do not undergo migration and do not influence the transport of any charged species. Such a situation can be encountered in electrode reactions involving, e.g., metallomacrocycles, yielding a small ion (a metal ion or $\mathrm{H}^{+}$ ion) and a large neutral organic moiety. The ratio of diffusion coefficients of the reactant and the charged product can then even exceed one order of magnitude. The plot of the limiting current in the case of the charge-decrease reaction looks very similar to that of
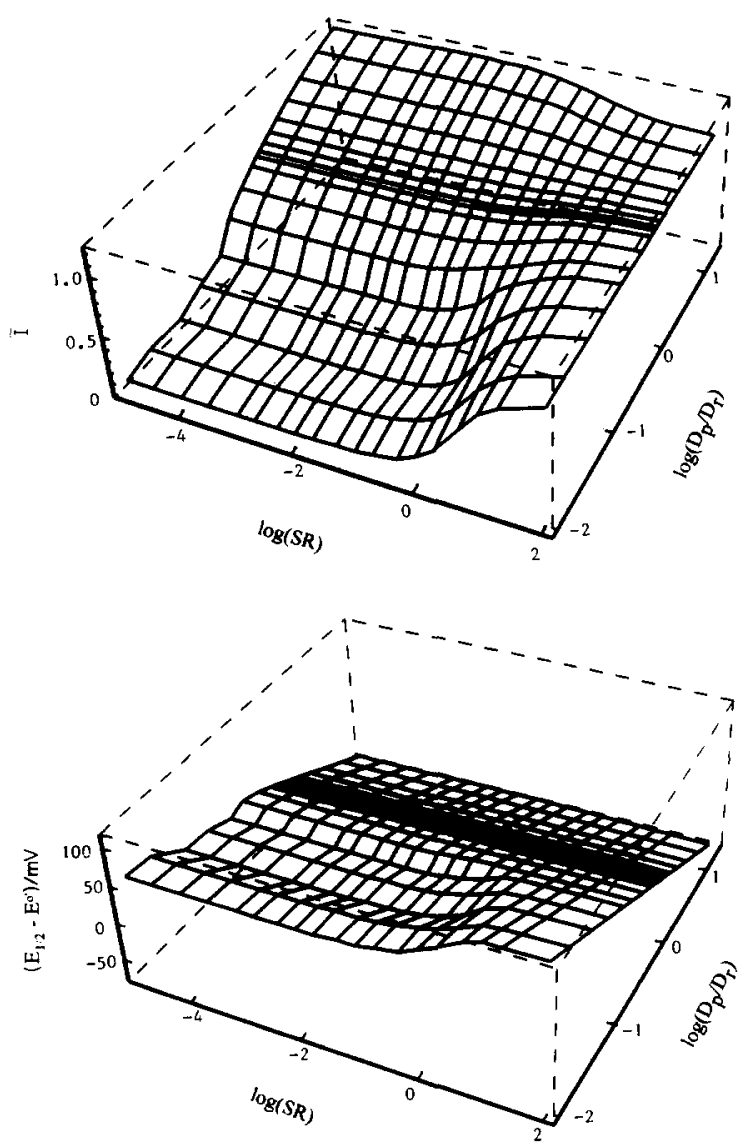

Fig. 9. Dependence of limiting current and the half-wave potential of steady-state $I-E$ curve on the ratio of supporting electrolyte and reactant concentrations and on the ratio of diffusion coefficients of the product and the reactant. Case: charge increase. Simulation conditions: $R_{\max }=1200$, first $\delta \rho=0.01, N_{x}=6, \partial T=1.0 \times 10^{-9}$, step in $I$ value $=0.01$. Diffusion coefficients of supporting electrolyte ions assumed to be equal to the diffusion coefficient of the reacting ion.
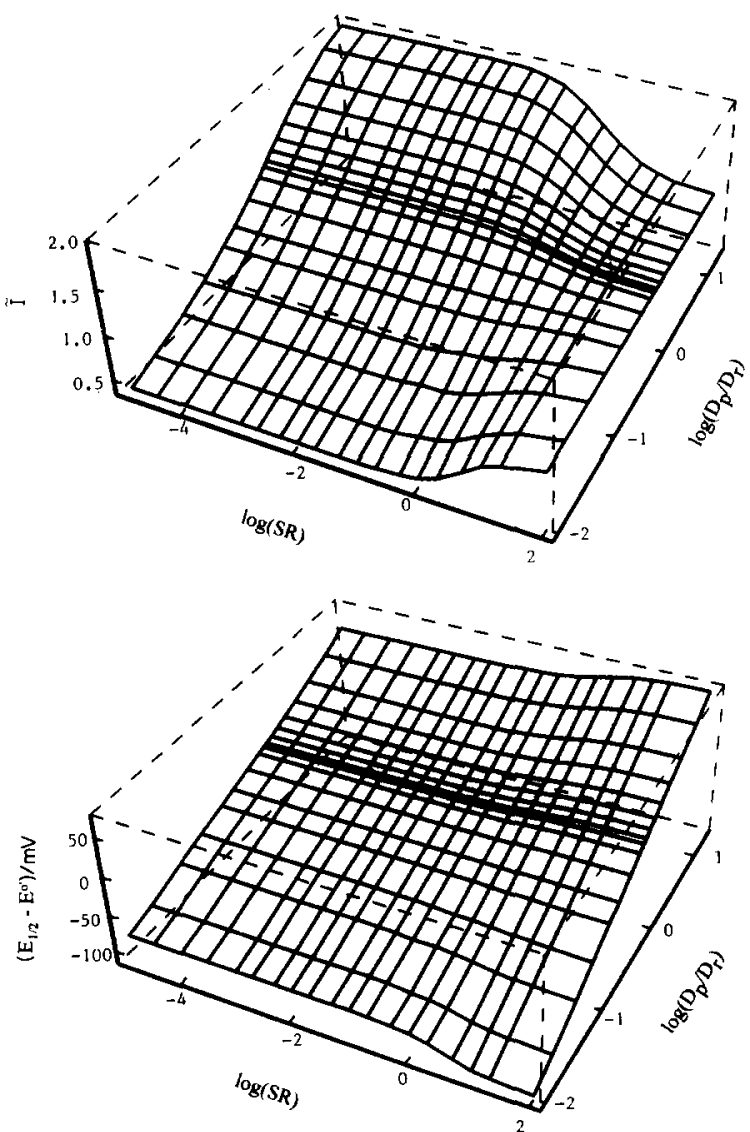

Fig. 10. Dependence of limiting current and the half-wave potential of steady-state $I-E$ curve on the ratio of supporting electrolyte and reactant concentrations and on the ratio of diffusion coefficients of the product and the reactant. Case: charge decrease. Simulation conditions: $R_{\max }=1200$, first $\delta \rho=0.01, N_{x}=6, \partial T=1.0 \times 10^{-9}$, step in $I$ value $=0.01$. Diffusion coefficients of supporting electrolyte ions assumed to be equal to the diffusion coefficient of the reacting ion.

the charge-increase process. The Myland-Oldham theory predicts a moderate increase of the current when the support ratio decreases. Fig. 10 shows that when the diffusion coefficient of the product is very high, the increase of the current is much above that predicted for equal diffusion coefficients, and the mechanism of this elevation is the same as in the previously discussed case: the fast diffusional removal of the product is compensated (partially) by the higher transport rate of the reactant.

When the diffusion coefficient of the product is lower than that of the reactant, the product is accumulated in the neighbourhood of the electrode, impeding the transport of the reactant. When the diffusion coefficient ratio becomes very small, the product accumulation can suppress the limiting current almost entirely.

In the third case (charge-cancellation reaction), no significant change of the limiting current value is observed when the diffusion coefficients ratio varies. This effect can be expected, because the uncharged product is transported solely by diffusion and the change of the 

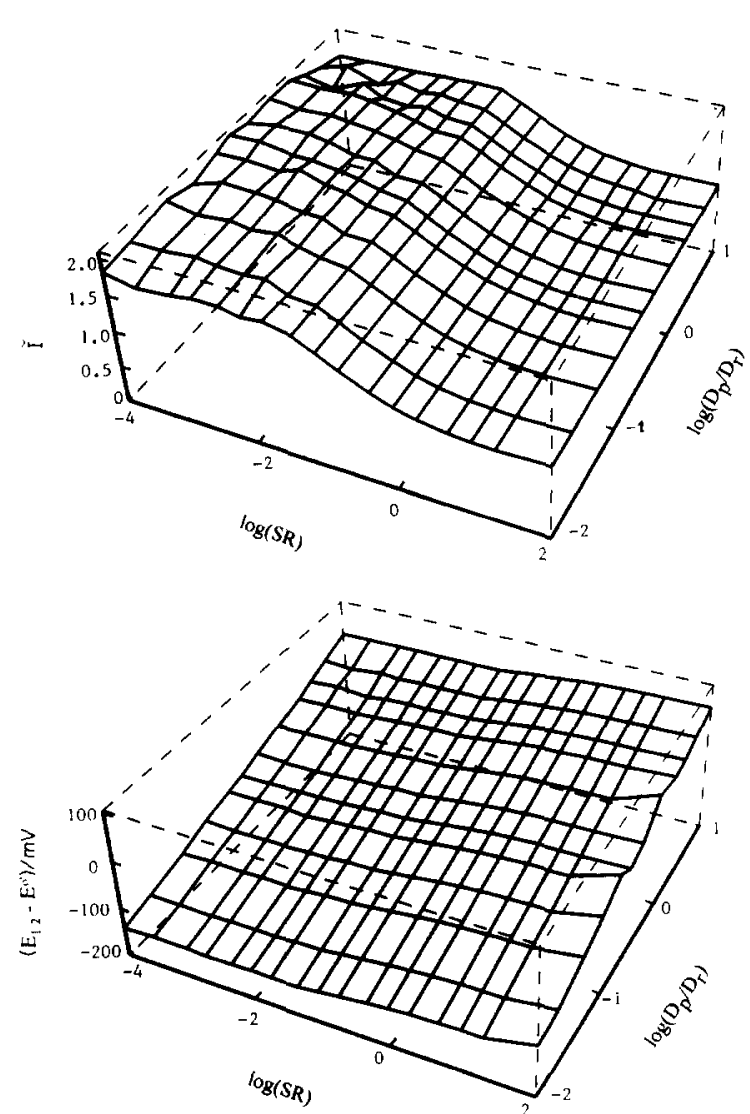

Fig. 11. Dependence of limiting current and the half-wave potential of steady-state $I-E$ curve on the ratio of supporting electrolyte and reactant concentrations and on the ratio of diffusion coefficients of the product and the reactant. Case: charge cancellation. Simulation conditions: $R_{\max }=1200$, first $\delta \rho=0.01, N_{x}=6, \partial T=1.0 \times 10^{-9}$, step in $I$ value $=0.01$. Diffusion coefficients of supporting electrolyte ions assumed to be equal to the diffusion coefficient of the reacting ion.

diffusion coefficient does not influence the transport of any other species, including the reactant. Fig. 11 covers support ratio values down to $10^{-4}$ and, despite the fact that the results obtained for very low SR are not as reliable as for high SR, the general trend is clear: the current increases with decrease in the SR and levels off for a support ratio close to zero.

Variations of the half-wave potentials in all these three cases show a regular pattern: generally, a linear shift of $E_{1 / 2}$ with the logarithm of the diffusion coefficient ratio can be observed. In regions where the normalized limiting current differs significantly from unity, additional variation of the half-wave potential can be seen. The first variation pattern is a usual change of the ratio of reactant and product concentration at the electrode surface if their diffusions are not equal, observed also when the excess of the supporting electrolyte is present. The second pattern reflects changes in surface concentrations of the reactant and the product due to the migrational component in the transport.

\subsection{More complicated electrode reactions}

For more complicated electrode reactions, the number of variable parameters becomes so large that exhaustive analysis is not feasible. Therefore, we show results obtained for only one example:

$\mathrm{X}^{+}+\mathrm{Y}^{-} \rightarrow \mathrm{Z}^{+}+\mathrm{e}^{-}$

for various support ratios (Fig. 12). It can be seen that both concentrations of the reactants and their diffusion coefficients influence either the position of the wave or its height. The rather weak effect of the change in the support ratio can be explained by the relatively high total concentration of ions (both reactants are ionic) and a good conductivity of the solution.

\subsection{Generalizations}

On the basis of the results presented, it is possible to make two general predictions. If the reaction scheme involves only one ionic reactant and one ionic product with the same sign, the accumulation of the product near the electrode (low $D_{\mathrm{p}} / D_{\mathrm{r}}$ ratio) will suppress the current whereas a high $D_{\mathrm{p}} / D_{\mathrm{r}}$ value will increase it. If this ratio differs significantly from unity, the type of the reaction (charge increase or charge decrease) does not matter.

All reactions involving one reactant and yielding a number of products, of which only one is charged, have
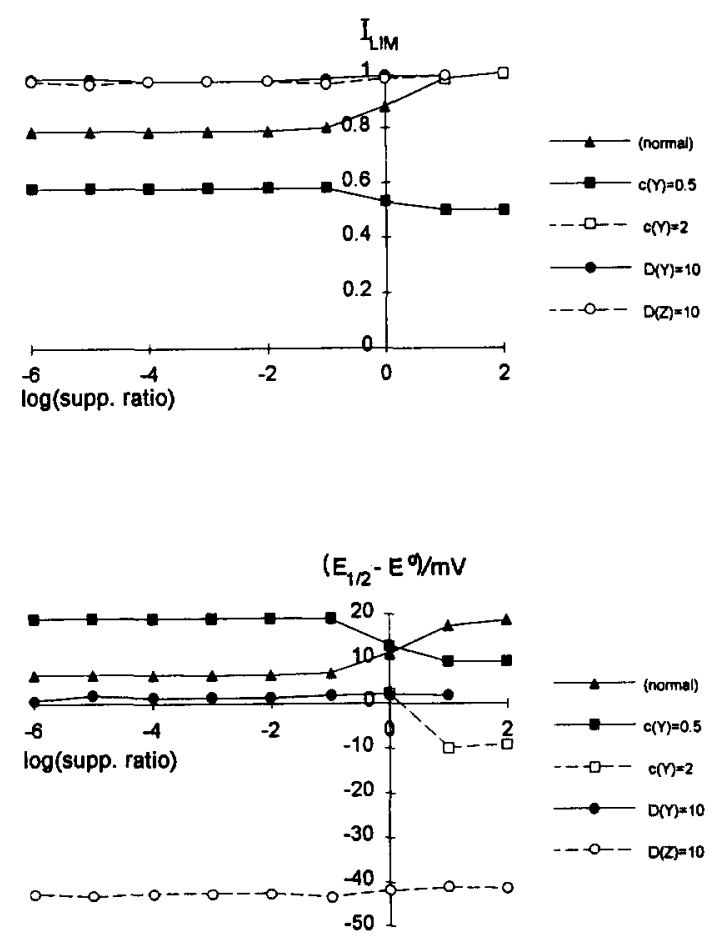

Fig. 12. Plots of limiting current and half-wave potential dependence on the support ratio for reaction (17). Normal simulation conditions: $R_{\max }=1200$, first $\delta \rho=0.01, \delta T=1.0 \times 10^{-9}, N_{x}=6$, all diffusion coefficients equal, concentrations of both reactants equal. 
the same characteristics as reactions leading only to the charged product, because neutral products do not affect the transport of any charged species. Although the results presented were obtained for hemispherical electrodes, they should be qualitatively valid for disc geometries also, provided that the redox system exhibits nernstian behavior.

\section{Conclusions}

The results obtained lead to the conclusion that the simulation method presented offers a reliable and versatile tool for modelling systems characterized by mixed diffusional and migrational transport to a hemispherical microelectrode, in the absence of double-layer effects. In contrast to the existing methods, our approach imposes no limitations on the values of the diffusion coefficients and no limitations on the number of species involved or produced in the electrode reaction. The concentration profiles can be used to construct chronopotentiograms and steady-state $I-E$ curves for any value of the electron transfer rate. Therefore it can be expected that our method will be better suited for the examination and interpretation of real experimental situations. These include, among others, investigation of complex formation reactions and equilibria and, e.g., studies of molecular receptors. The application to studies of complexation equilibria will be the subject of a forthcoming publication.

The developed simulation scheme has a very good performance for charge-increase and charge-decrease reactions, irrespective of the support ratio value. The performance for other types of reactions becomes unsatisfactory when the support ratio is lower than 0.01 and the imposed current is close to the limiting current (the top part of the wave). For all situations where the support ratio is higher than 0.01 , the performance of the simulation is very good.

From the results presented, it can be concluded that the often neglected influence of diffusion coefficients on the steady-state $I-E$ curves obtained under migrational conditions can be significant, in some situations leading even to the inversion of the trends in the electric variables that are predicted on the basis of simpler models.

\section{Acknowledgements}

This work was supported in part by a grant (BST$439 / 5 / 93$ ) from the University of Warsaw. The com- putation time provided by the Laboratory of Quantum Chemistry, the Interdisciplinary Centre of Mathematical and Computational Modelling (ICM) and the Department of Mathematics and Informatics of the University of Warsaw is gratefully acknowledged.

\section{References}

[1] M. Fleischmann, S. Pons, D.R. Rolison and P. Schmidt (eds.), Ultramicroelectrodes, Datatech, Morganton, NC, 1987.

[2] Microelectrodes: Theory and Application, NATO ASI Series, Vol. 197, Kluwer, Dordrecht, 1991.

[3] M. Ciszkowska, Z. Stojek, S.E. Morris and J.G. Osteryoung, Anal. Chem., 64 (1992) 2372.

[4] S. Daniele and G.A. Mazzocchin, Anal. Chim. Acta, 273 (1993) 3.

[5] L. Nyholm and G. Wikmark, Anal. Chim. Acta, 273 (1993) 41.

[6] M. Ciszkowska and Z. Stojek, J. Electroanal. Chem., 344 (1993) 135.

[7] M. Ciszkowska and Z. Stojek, Analyst (London), 119 (1994) 239.

[8] C. Amatore, M.R. Deakin and R.M. Wightman, J. Electroanal. Chem., 255 (1987) 49.

[9] C. Amatore, J. Bartelt, M.R. Deakin and R.M. Wightman, J. Electroanal. Chem., 256 (1988) 255.

[10] K.B. Oldham, J. Electroanal. Chem., 250 (1988) 1.

[11] K.B. Oldham, J. Electroanal. Chem., 337 (1992) 91.

[12] J.B. Cooper and A.M. Bond, J. Electroanal. Chem., 315 (1991) 143.

[13] J.B. Cooper, A.M. Bond and K.B. Oldham, J. Electroanal. Chem., 331 (1992) 877.

[14] D.R. Baker, M.W. Verbrugge and J. Newman, J. Electroanal. Chem., 314 (1991) 23.

[15] J.C. Myland and K.B. Oldham, J. Electroanal. Chem., 347 (1993) 49.

[16] J.D. Norton, H.S. White and S.W. Feldberg, J. Phys. Chem., 94 (1990) 6772.

[17] J.D. Norton, W.E. Benson, H.S. White, B.D. Pendley and H.D. Abruna, Anal. Chem. 63 (1991) 1909.

[18] W. Kemula and M. Michalski, Rocz. Chem., 16 (1936) 535.

[19] J. Heyrovsky and J. Kùta, Grundlagen der Polarographie, Akademie-Verlag, Berlin, 1965, p. 53.

[20] Yu. I. Kharkats and A.V. Sokirko, J. Electroanal. Chem., 303 (1993) 27.

[21] C.P. Smith and H.S. White, Anal. Chem. 65 (1993) 3343.

[22] D. Britz, Digital Simulation in Electrochemistry, Springer, Berlin, 1988.

[23] J. Crank and P. Nicolson, Proc. Cambridge Philos. Soc., 43 (1947) 50.

[24] J. Heinze, M. Störzbach and M. Mortensen, J. Electroanal. Chem., 165 (1984) 61.

[25] S.W. Feldberg, J. Electroanal. Chem., 127 (1981) 1.

[26] W.H. Press, B.P. Flannery, S.A. Teukolsky and W.T. Vetterling, Numerical Recipes, Cambridge University Press, Cambridge, 1989 , p. 49.

[27] G. Mamantov and P. Delahay, J. Am. Chem. Soc., 76 (1954) 5323. 